FACTA UNIVERSITATIS

Series: Mechanical Engineering Vol. 17, No 1, 2019, pp. 65 - 74

https://doi.org/10.22190/FUME190120009W

Original scientific paper

\title{
SYNERGISTIC TRIBOLOGICAL PROPERTIES OF SYNTHETIC MAGNESIUM SILICATE HYDROXIDE COMBINED WITH AMPHIPHILIC MOLECULES
}

\section{Bin Wang, Qiu Ying Chang, Kai Gao}

School of Mechanical, Electronic and Control Engineering, Beijing Jiaotong University, Beijing, PR China

\begin{abstract}
This paper reports the synthesis of magnesium silicate hydroxide (MSH) nanoparticles and their synergistic tribological properties combined with amphiphilic molecules (AMs) as additives in base oil. This combination reduces wear losses substantially due to the formation of a double well-arranged molecular layer or tribofilm on the rubbing surfaces under certain test conditions. From the results of nonequilibrium molecular dynamics (NEMD) simulations, lamellate MSH nanoparticles provide a medium for the adsorption of AMs thus further decreasing the contact of rough peaks. In addition, with the increase of load, a tribofilm containing element $\mathrm{Mg}$, Si, O forms on the worn surfaces and greatly improves the anti-wear property of base oil.
\end{abstract}

Key Words: Synthetic Magnesium Silicate Hydroxide; Amphiphilic Molecules; Friction and Wear; Nonequilibrium Molecular Dynamic Simulation

\section{INTRODUCTION}

To obtain more excellent tribological properties, a variety of approaches including new wear resistant materials [1,2], coating [3], new lubricant additives [4-6], various low-viscosity base oil and others have been explored widely. Among these, finding out new additives with outstanding tribological and dispersive properties which can replace or partly replace the traditional lubricant additives such as zinc dithiophosphate (ZDDP) [7], is always the focus of research.

Magnesium silicate hydroxide (MSH) is a new type of lubricant additive with an ideal chemical formula of $\mathrm{Mg}_{3} \mathrm{Si}_{2} \mathrm{O}_{5}(\mathrm{OH})_{4}$. Because of the weaker Van der Waals forces and hydrogen bonds between $\mathrm{Si}-\mathrm{O}$ tetrahedral sheet and $\mathrm{Mg}-\mathrm{O} / \mathrm{OH}$ octahedral sheet, it is easy to

Received January 20, 2019 / Accepted March 15, 2019

Corresponding author: Bin Wang

Affiliation: School of Mechanical, Electronic and Control Engineering, Beijing Jiaotong University

E-mail: 15116341@bjtu.edu.cn 
decompose and release unsaturated groups such as $\mathrm{Si}-\mathrm{O}-\mathrm{Si} 、 \mathrm{O}-\mathrm{Si}-\mathrm{O} 、 \mathrm{OH}-\mathrm{Mg}-\mathrm{OH}(\mathrm{O})$ 、 $\mathrm{OH}^{-}$and $\mathrm{O}-\mathrm{H}-\mathrm{O}$ [8-12]. Actually, because of these features, scholars have systematically investigated the anti-wear behavior of serpentine powders whose composition is mainly MSH, as additives in oil under different friction conditions and concentrations [13-15]. The results show that a tribofilm mainly consisting of $\mathrm{Fe}, \mathrm{C}, \mathrm{O}$ and $\mathrm{Si}$ elements forms on the rubbing surfaces, which indicates that serpentine particles decomposed under the combination of local high pressure and temperature. However, as a type of natural mineral, serpentine contains a small quantity of aluminum oxide, calcium oxide, iron oxide and other compounds which work against the clarification of its tribological mechanisms. Meanwhile, by the method of high-energy mechanical ball-milling, only micro-sized natural serpentine particles can be obtained, which is not suitable for their applications as lubricant additives. Inspired by the above research studies, we have synthesized MSH nanoparticles and confirmed its outstanding tribological properties in base oil [16-18].

In order to disperse the nanoparticles uniformly and steadily in oil, amphiphilic molecules (AMs) like oleic acid or stearic acid are commonly used as a modification agent [19-21]. These molecules do not only adsorb around the nanoparticles and prevent the agglomeration effectively, but also reduce friction and wear by itself [5]. Early in 1920s, organic friction modifiers (OFMs) based on AMs were introduced to increase the energy efficiency of equipment, and are always one of the most important additives in lubricant oil. The main friction-reducing mechanism of OFMs is that it can form a vertically oriented, close-packed monolayer on the sliding surfaces [22-24]. To our knowledge, although there have been many literatures on the surface modification effects and the friction properties of OFMs, few research studies have been focused on the synergistic tribological behaviors of nanoparticles combined with AMs. So as to improve the dispersity of natural serpentine particles in oil, Xu et al. [13-15] used oleic acid or other AMs as a surface modifier during the process of ball-milling and studied the tribological performance of these surface-coated particles without considering the anti-wear effect of AMs. Song et al [21] prepared surface-modified $\mathrm{ZnAl}_{2} \mathrm{O}_{4}$ nanoparticles by heating and drying a solution containing particles and oleic acid, then also tested their tribological properties by ignoring the influence of oleic acid.

In this paper, we synthesized lamellate MSH nanoparticles hydrothermally and explored their synergistic tribological behaviors combined with amphiphilic organic molecules while improving its dispersity in polyalphaolefin base oil (PAO). At the same time, techniques of nonequilibrium molecular dynamics (NEMD) simulations, scanning electron microscopy (SEM) and energy dispersive spectroscopy (EDS) were used to explain the anti-wear mechanisms.

\section{EXPERIMENT AND MATERIALS}

The synthesis of MSH nanoparticles was carried out in autoclaves by the method of hydrothermal reaction. Nano-sized magnesium oxide $(\mathrm{MgO},>99.9 \mathrm{wt} \%, 40 \mathrm{~nm})$ and silicon dioxide $\left(\mathrm{SiO}_{2},>99.9 \mathrm{wt} \%, 40 \mathrm{~nm}\right)$ were used as precursors and their reaction concentrations were $2.7 \times 10^{-7} \mathrm{~mol} / \mathrm{L}$ and $1.8 \times 10^{-7} \mathrm{~mol} / \mathrm{L}$, respectively. The mixture was hydrothermally treated in a reactor containing $\mathrm{NaOH}$ aqueous solution at temperature of $200^{\circ} \mathrm{C}$, pressure of $1.6 \mathrm{MPa}$ and experiment duration of $12 \mathrm{~h}$. The resulting powders were washed in distilled water for three times to remove sodium and dried in furnace at $80^{\circ} \mathrm{C}$ for $10 \mathrm{~h}$. Table 1 lists 
the X-ray Fluorescence Spectrometer (XRF) result of the synthetic MSH particles. Its crystal formula can be expressed as $\mathrm{Mg}_{2.329} \mathrm{Si}_{2.202} \mathrm{Na}_{0.469} \mathrm{O}_{5}(\mathrm{OH})_{4}$. The morphology of the synthetic MSH nanoparticles was investigated by SEM in Fig. 1.

Table 1 Chemical elements of synthetic MSH nanoparticles

\begin{tabular}{lr}
\hline Elements & Content (wt \%) \\
\hline Magnesium (Mg) & 24.19 \\
Silicate (Si) & 26.72 \\
Sodium (Na) & 2.23 \\
\hline
\end{tabular}

Synthetic MSH nanoparticles were ultrasonically dispersed in PAO base oil with a viscosity of $73 \mathrm{cSt}$ at $40^{\circ} \mathrm{C}$ and using $\mathrm{AMs}$ as a dispersant agent (termed as oil+ MSH+ AMs). The weight percentage of the powder and AMs in the oil-powder suspension was $1 \%$ and $2 \%$, respectively. To make clear the influence of AMs on the tribological performance of additives, pure oil only added $2 \mathrm{wt} \%$ AMs (termed as oil+ AMs) also prepared at the same time.

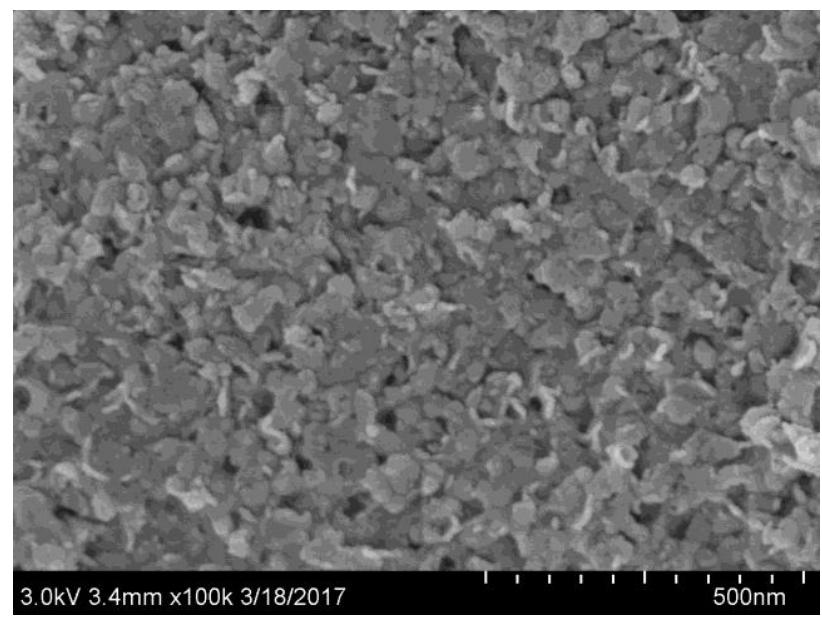

Fig. 1 SEM morphology of synthetic MSH nanoparticles.

Tribological experiments were carried out by a four-ball friction and wear test machine (MRS-10A). GCr15 balls with 62 67 HRC hardness and $0.02 \mu \mathrm{m}$ roughness (Ra) were used. The radius, Poisson's ratio and Young's modulus of ball specimens are $6.35 \mathrm{~mm}, 0.3$ and 208GPa, respectively. The experimental conditions were: normal loads 200 and $600 \mathrm{~N}$ (corresponding to maximum pressures of 2.71 and $3.91 \mathrm{GPa}$, respectively), rotational speeds 400rpm (corresponding to speeds of $0.153 \mathrm{~m} / \mathrm{s}$ ), duration two hours at room temperature. Once the test was finished, the wear scar diameter of ball was obtained by using an optical microscope (accuracy is $0.01 \mathrm{~mm}$ ) and all sets of the experiment were repeated three times. The morphologies and chemical elements of the worn surfaces were characterized by SEM and EDS. 


\section{RESULTS AND DISCUSSION}

\subsection{Lubricant additives}

From Fig. 1, we can see that the synthetic MSH nanoparticles are mostly lamellate and have an average lateral dimension of approximately $50 \mathrm{~nm} \times 10 \mathrm{~nm}$. There are also some MSH nanoparticles curled to a certain extent, which is attributed to the hydrothermal conditions. And specific synthesis mechanism of MSH can refer to our previous study [17].

\subsection{Dispersion property}

To illustrate the influence of AMs on the dispersive property of synthetic MSH nanoparticles in oil, sedimental tests lasted 7 days from oil sample preparation were carried out (Fig. 2). AMs make MSH nanoparticles dispersed in oil homogeneously and stably, however, a layer of sediment at the bottom of bottle was formed when only added MSH nanoparticles in oil (Fig. 2(b)).

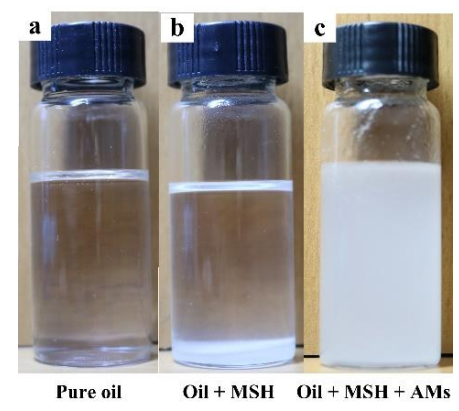

Fig. 2 Oil-additive suspensions after 7 days from preparing (a) pure oil and (b) oil only added MSH and (c) oil added both MSH and AMs. In this sedimental test, pure oil only added synthetic MSH nanoparticles with a weight percentage of $2 \%$ (termed as oil+ MSH) prepared at the same time

\subsection{Friction and wear}

Fig. 3 shows the average wear scar diameters (WSDs) and the three-dimensional profiles of samples experimented in three oil samples under different conditions. Obviously, the anti-wear property of base oil was significantly improved by the addition AMs, and better results were further obtained after suspending synthetic MSH nanoparticles in oil under both experimental conditions. With respect to the average WSDs, the anti-wear rates of oil+ MSH+ AMs relative to pure oil were $45.56 \%$ and $32.44 \%$ under $200 \mathrm{~N}$ and $600 \mathrm{~N}$, respectively. However, it can be seen from Fig. 3 (b) that there happened precious little wear when used synthetic MSH as additives in oil, meanwhile, the volume wear rate of friction specimens subjected to oil+ MSH+ AMs decreased by the order of magnitude with respect to that of specimens lubricated by pure base oil or oil+ AMs. Under the test condition of $200 \mathrm{~N}$ and $400 \mathrm{rpm}$, the average WSDs of $285.8 \mu \mathrm{m}$ is close to the Hertz contact diameter of $240 \mu \mathrm{m}$ calculated by Hertz contact radius formula (Eq. (1)) which also means little wear happened when lubricated by oil+ MSH+ AMs. This is because the four-ball friction and wear tests are based on a point contact mode, and the contact pressure is high to 


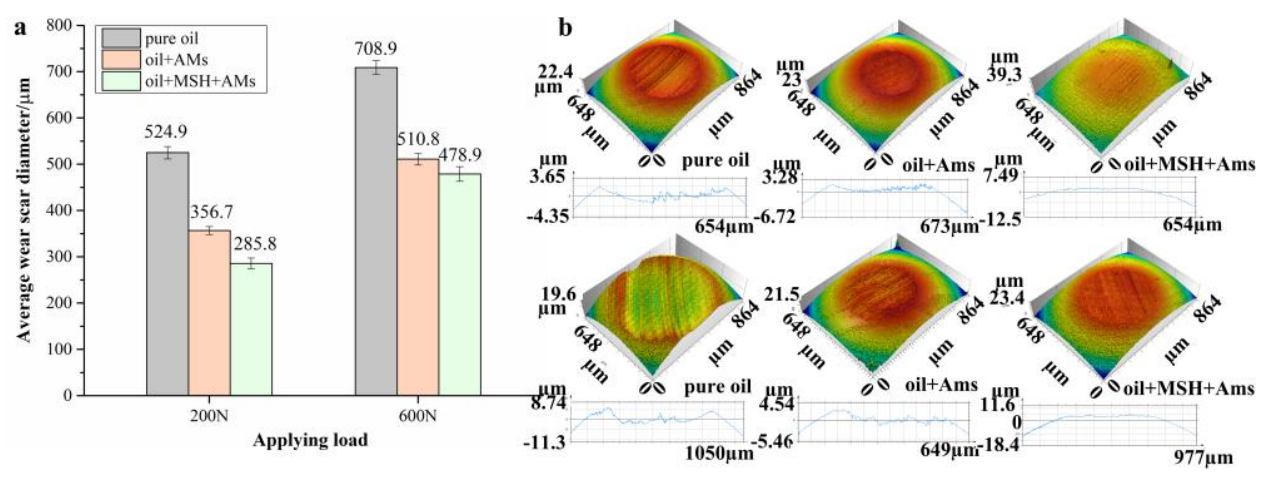

Fig. 3 Average wear scar diameter values (a) and the three-dimensional profiles (b) of samples tested in pure base oil, oil+ AMs and oil+ MSH+ AMs under different test conditions

gigapascals level attributed to the small lubrication area. Therefore, the surface deformation cannot be ignored. Hertz contact theory is one of the starting points of electrohydrodynamic lubrication (EHL) theory considering the elastic deformation of the surface. In our study, it is likely that the ball material will be removed due to the maximum experimental pressure of 2.91 or $3.71 \mathrm{GPa}$, and the wear scar diameter is greater than that of Hertz contact diameter. Besides, the D-value between them can be used to evaluate the oil's anti-wear property, that is, the smaller the difference, the higher the anti-wear.

$$
a=\sqrt[3]{\frac{3 F}{4} \frac{\frac{1-\mu_{1}^{2}}{E_{1}}+\frac{1-\mu_{2}^{2}}{E_{2}}}{\frac{1}{\rho_{1}}+\frac{1}{\rho_{2}}}}
$$

Among them, $a$ is the Hertz contact radius; $F$ is the applied load; $\mu_{1}$ and $\mu_{2}$ are the Poisson's ratio of ball specimens; $E_{1}$ and $E_{2}$ are the Young's modulus of ball specimens; $\rho_{1}$ and $\rho_{2}$ are radius of ball specimens.

Although the coefficient of friction (COF) value obtained by the four-ball friction and wear machine cannot be used seriously to explore the friction property of oil, the variation trend of COF with different oil samples under same conditions is of great significance. In this study, all sets of the experiment were repeated three times, and Fig. 4a shows the average COF value during friction tests for samples lubricated with different oil samples. Under the condition of $200 \mathrm{~N}$, if the error is considered, there is no significant difference in COFs of samples lubricated in pure oil, oil+ AMs and oil+ MSH+ AMs. With the load increased to $600 \mathrm{~N}$, oil only added AMs also had similar stable value of COF than that of oil added synthetic MSH particles. However, pure oil has a lower average stable COF value of 0.062. To further analyze the friction property of three different oil samples, Figs.4b and c shows the evolution of the COF for one of the three repeated friction tests under experimental conditions of $200 \mathrm{~N}$, 400rpm and 600N, 400rpm. Both in Figs. $4 \mathrm{~b}$ and c, at the loading stage, the COFs of the samples lubricated in pure oil increased first and then decreased 

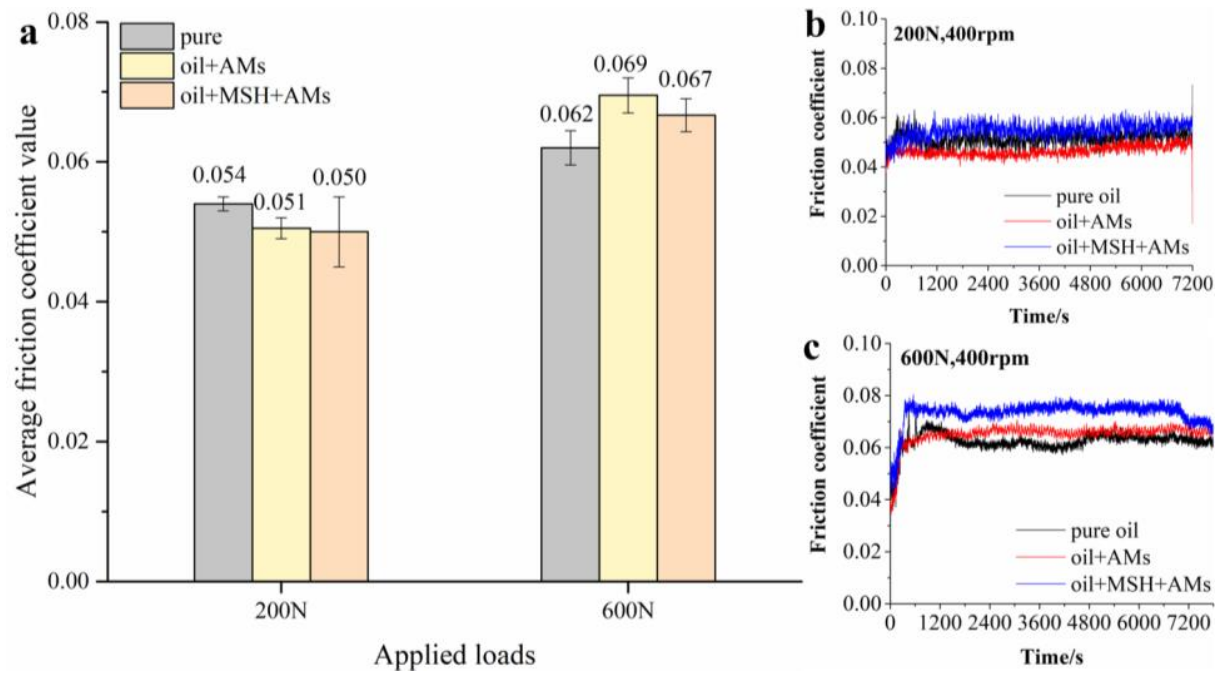

Fig. 4 (a) Average coefficient of friction (COF) value, and (b, c) evolutions COF during friction and wear tests for balls lubricated with pure base oil, oil+ AMs and oil+ MSH+ AMs under experimental condition of (b) $200 \mathrm{~N}$, 400rpm and (c) $600 \mathrm{~N}$, $400 \mathrm{rpm}$. Because the load of $600 \mathrm{~N}$ was imposed stepwise from $200 \mathrm{~N}$ with a step of $100 \mathrm{~N}$ every $2.5 \mathrm{~min}$, the experimental time in Fig. $4 \mathrm{c}$ is longer than that in Fig. $4 \mathrm{~b}$

which means there happened severe wear and the actual contact area increased to a certain extent. In other words, the higher stable COFs of samples lubricated in oil+ MSH+ AMs do not mean oil containing MSH nanoparticles have worse friction-reduction property than that of pure oil, and it may be attributed to the distinctive frictional performance of tribofilm formed on worn surfaces.

Fig. 5 shows the SEM morphologies of worn surfaces lubricated with base oil and oil+ MSH+ AMs under different loads. There are a great amount of scratches and furrows on the surfaces for pure base oil, which indicates severe wear occurred on the contact surfaces under all experimental conditions. In contrast, the furrows become shallower and less when adding synthetic MSH nanoparticles to oil. The sliding surfaces are extraordinary smooth under the experimental condition of $200 \mathrm{~N}$, which is in accordance with the anti-wear results in Fig. 3. Meanwhile, a dark tribofilm formed on the substrate surface when the experimental load increased to $600 \mathrm{~N}$.

In Fig. 6, the EDS analyses of friction surfaces lubricated by oil+ MSH+ AMs show that the smooth worn surfaces under test condition of $200 \mathrm{~N}$ mainly consist of $\mathrm{Fe}$ which agrees with EDS result of original substrate (Table 2). However, with the increase of experimental load to $600 \mathrm{~N}$, a dark tribofilm containing high content of $\mathrm{O}, \mathrm{Mg}$ and $\mathrm{Si}$ elements formed on the sliding surfaces. $\mathrm{Mg}$ and $\mathrm{Si}$ elements come from synthetic MSH additives. Meanwhile, the chemical compositions (Table 2) reveal that the molar rates between $\mathrm{Mg}$ and $\mathrm{Si}$ are different from that of synthetic MSH nanoparticles, indicating a decomposition of MSH occurred under the combination of mechanical and thermal energy during the tribological tests. 


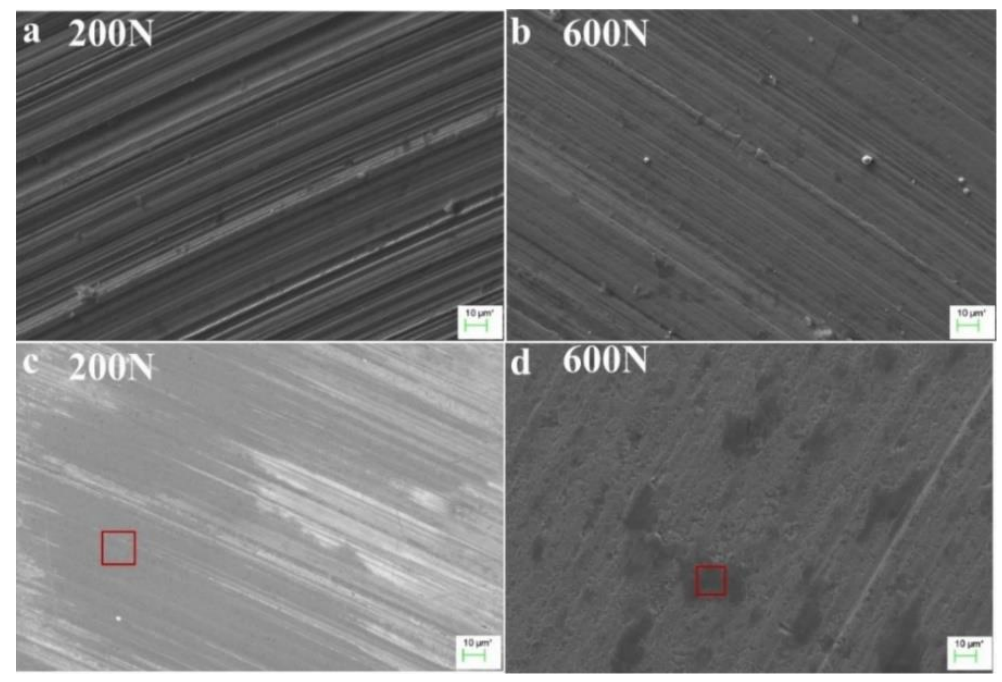

Fig. 5 SEM morphologies of the worn surfaces lubricated with (a) (b) pure oil and (c) (d) oil $+\mathrm{MSH}+\mathrm{AMs}$ under experimental conditions of $200 \mathrm{~N}$ and $600 \mathrm{~N}$ (The red frames in this figure are the component analysis areas of EDS)
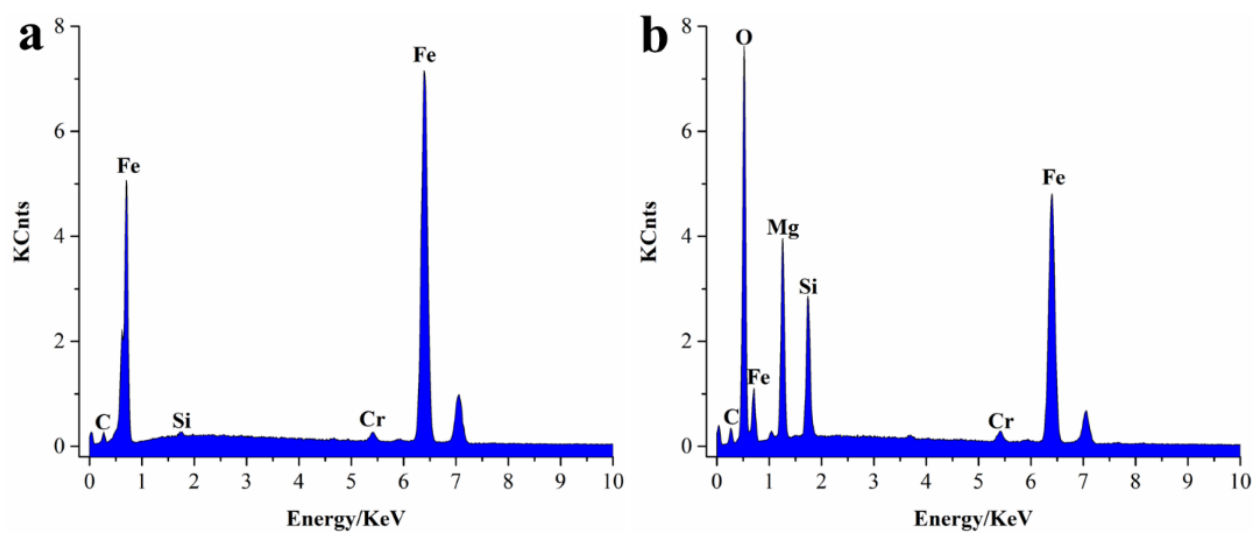

Fig. 6 EDS spectra of the worn surfaces (marked areas in Fig. 5) lubricated with oil added both MSH and AMs under experimental conditions of (a) $200 \mathrm{~N}$ (b) $600 \mathrm{~N}$

Table 2 Chemical compositions of the worn surfaces for different experimental conditions

\begin{tabular}{lcccc}
\hline \multirow{2}{*}{ Samples } & Content (at \%) & & \\
\cline { 2 - 5 } & $\mathrm{Fe}$ & $\mathrm{O}$ & $\mathrm{Mg}$ & $\mathrm{Si}$ \\
\hline Original substrate & 82.3 & - & - & 0.3 \\
Smooth area in Fig.5c & 78.0 & 0.2 & - & 0.6 \\
Dark tribofilm in Fig. 5d & 20.9 & 45.8 & 13.4 & 6.1 \\
\hline
\end{tabular}




\subsection{Nonequilibrium molecular dynamic simulations}

To fully understand the performance of lubricant molecule and additives, NEMD simulation including initial configuration composed of hexadecane molecules, stearic acid monolayers, and lamellate iron nanocluster confined between smooth steels was carried out. (100) surface of $\alpha$-iron, stearic acid molecules and iron nanocluster were used as sliding tribo-surfaces, AMs, and nanoparticles, respectively. Although silicate would be a more accurate representation of an additive in this system, there is no classical MD force-field. This size domain was optimized considering the effects of itself and the simulation time. A brief explanation of simulation setup and snapshots after $300 \mathrm{ps}$ of sliding are shown in Fig. 7. Periodic boundary conditions were applied in the $\mathrm{x}$ and $\mathrm{y}$ directions with size of $31.53 \times 31.53 \AA^{2}$ and $\mathrm{z}$ dimension varied from $94 \sim 135 \AA$ according to the initial setup of models. Both simulations were performed at $353 \mathrm{~K}$ which is representative of experimental temperature and controlled by a Langevin thermostat. The density of lubricant liquid was $0.76 \mathrm{~g} / \mathrm{cm}^{3}$ and the surface coverage of stearic acid on all surfaces was $4.5 \mathrm{~nm}^{-2}$. The applying load and shear rate were $3 \mathrm{GPa}$ and $10 \mathrm{~m} / \mathrm{s}$, respectively. All-atom force fields were used in the NEMD simulations which enables the structure and large molecular systems to be reliably analyzed. COMPASS force field was applied for hexadecane and AMs molecules, and Embedded Atom Model (EAM) potential was represented the iron-iron interactions within the slabs. The Lennard-Jones (LJ) potential with cut-off distance of $12.5 \AA$ was used for van der Waals and long-range Columbic interactions between the lubricant and the surfaces. The potential parameters can refer to
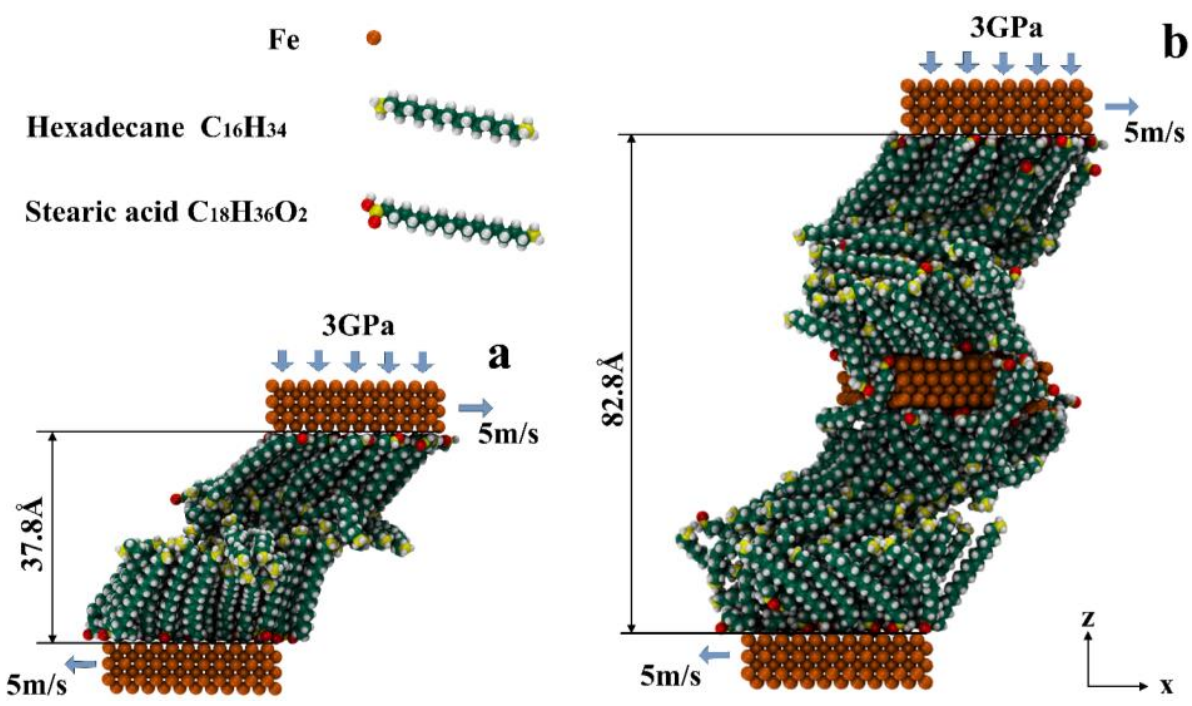

Fig. 7 Simulation setup and snapshots after 300ps of sliding (a) confined three layers of hexadecane molecules in between two stearic acid monolayers adsorbed on (100) surfaces of $\alpha$-iron (b) confined three layers of hexadecane molecules in between each pair of stearic acid monolayers adsorbed on (100) surfaces of $\alpha$-iron and lamellate iron nanocluster. $\mathrm{Fe}, \mathrm{C}, \mathrm{O}$, and $\mathrm{H}$ atoms are presented as orange, cyan (terminal $\mathrm{C}$ in yellow), red, and white colors, respectively 
literatures $[25,26]$. The simulation procedure can be divided into three main stages: i) optimization of structure, ii) compression in $\mathrm{z}$ direction under applying load of $3 \mathrm{Gpa}$, and iii) friction and wear in $\mathrm{x}$ direction with shear rate of $10 \mathrm{~m} / \mathrm{s}$. A time relaxation constant of $0.1 \mathrm{fs}$ was used and total simulations time was 550ps.

Under the confinement and sliding motion of the slabs, stearic acid molecules formed a vertically arranged solid-like layer on both surfaces of slab and iron nanocluster which can improve the bearing capacity of base oil to a great extent [22, 27-29]. The thickness of oil added stearic acid and oil added both stearic acid and flake iron cluster reduced from initial $55 \AA$ and $118 \AA$ to compressed $37.8 \AA$ and $82.8 \AA$, respectively. Lamellate particle in lubricating oil provided a medium to the adsorption of Ams; meanwhile, one more pair of vertical arranged layers would be formed between the sliding surfaces as shown in Fig.7 b which isolated the friction surfaces completely to achieve "zero wear" (consistent with the three-dimensional profiles of test samples shown in Fig. 3b). However, with the increase of applying load or shearing velocity, these vertical arranged layers would be destroyed and lose their bearing capacity [22]. Under this situation, the features of nanoparticles and their tribological properties become substantially important.

In the NEMD simulation, an iron nanocluster was used as the representation of nano-additive but this does not mean any flake nano-material combined with AMs has the same anti-wear effect as MSH. Because of the unique constitution and layered structure, MSH nanoparticles are easy to spread and decompose under the condition of certain pressure and temperature meanwhile forming a tribofilm consisting of $\mathrm{Mg}, \mathrm{Si}$, and $\mathrm{O}$ on the friction surface instead of acting as abrasive particles. This tribofilm will serve as a secondary anti-wear protection once the lubricant molecules and AMs have been crushed.

\section{CONCLUSIONS}

In conclusion, we have investigated the synergistic tribological properties of synthetic lamellate MSH nanoparticles combined with AMs in PAO base oil. This combination not only makes nanoparticles dispersed in oil homogeneously but also improves the anti-wear property of base oil substantially. Under relatively slight conditions, lamellate MSH particles provide media for the adsorption of AMs to form arranged layers, thus reducing the contact of rough peaks effectively. On the other hand, with the increase of applying load, a tribofilm containing element $\mathrm{Mg}$, $\mathrm{Si}$ and $\mathrm{O}$ forms on the sliding surfaces and ensures a secondary anti-wear protection.

Acknowledgments: The authors would like to thank the National Natural Science Foundation of China (Grant No.51075026) and the National Defense Pre-Research Foundation of China (Grant No.H092013B001).

\section{REFERENCES}

1. Gershman, I., Gershman, E.I., Mironov, A.E., Fox-Rabinovich, G.S., Veldhuis, S.C.,2016, Application of the self-organization phenomenon in the development of wear resistant materials-A review, Entropy, 18(11), 385.

2. Lee, K., Hsu, J., Naugle, D., Liang, H., 2016, Multi-phase quasicrystalline alloys for superior wear resistance, Mater Des, 108, pp. 440-7.

3. Bhushan, B., Gupta, B.K., 1991, Handbook of Tribology: Materials, coatings, and surface treatments, McGraw-Hill, New York, United States. 
4. Ali, M.K.A., Xianjun, H., 2015, Improving the tribological behavior of internal combustion engines via the addition of nanoparticles to engine oils, Nanotechnol Rev, 4, pp. 347-58.

5. Gulzar, M., Masjuki, H.H., Kalam, M.A., Varman, M., Zulkifli, N.W.M., Mufti, R.A., et al, 2016, Tribological performance of nanoparticles as lubricating oil additives, J Nanoparticle Res, 18, pp. 1-25.

6. Dai, W., Kheireddin, B., Gao, H., Liang, H., 2016, Roles of nanoparticles in oil lubrication, Tribol Int, 102, pp. 88-98.

7. Rokosz, M.J., Chen, A.E., Lowe-Ma, C.K., Kucherov, A.V., Benson, D., Paputa Peck, M.C., et al., 2001, Characterization of phosphorus-poisoned automotive exhaust catalysts, Appl Catal B Environ, 33, pp. 205-15.

8. Mookherjee, M., Stixrude, L., 2009, Structure and elasticity of serpentine at high-pressure, Earth Planet Sci Lett, 279, pp. 11-9.

9. Veblen, D.R., Buseck, P.R., 1979, Serpentine minerals: intergrowths and new combination structures, Science, 206, pp. 1398-400.

10. Sclar, C.B., Carrison, L.C., Thomas, P., et al., 1966, High-pressure reaction and shear strength of serpentinized dunite, Science; 153 , pp. 1285-7.

11. Riecker, R.E., Rooney, T.P., 1966, Weakening of dunite by serpentine dehydration, Science, 152, pp. 196-8.

12. Stalder, R., Ulmer, P., 2001, Phase relations of a serpentine composition between 5 and 14 GPa: Significance of clinohumite and phase E as water carriers into the transition zone, Contrib to Mineral Petrol, 140, pp. 670-9.

13. Yu, H.L., Xu, Y., Shi, P.J., Wang, H.M., Zhao, Y., Xu, B.S., et al., 2010, Tribological behaviors of surface-coated serpentine ultrafine powders as lubricant additive, Tribol Int, 43, pp. 667-75.

14. Yu, H., Xu, Y., Shi, P., Wang, H., Wei, M., Zhao, K., et al., 2013, Microstructure, mechanical properties and tribological behavior of tribofilm generated from natural serpentine mineral powders as lubricant additive, Wear, 297, pp. 802-10.

15. Zhang, B., Xu, Y., Gao, F., Shi, P., Xu, B., Wu, Y., 2011, Sliding friction and wear behaviors of surface-coated natural serpentine mineral powders as lubricant additive, Appl Surf Sci, 257, pp. 2540-9.

16. Chang, Q., Rudenko, P., Miller, D.J., Wen, J., Berman, D., 2017, Tribology International Operando formation of an ultra-low friction boundary fi Im from synthetic magnesium silicon hydroxide additive, Tribiology Int, 110, pp. 35-40.

17. Wang, B., Chang, Q.Y., Gao, K., Fang, H.R., Qing, T., Zhou, N.N., 2018, The synthesis of magnesium silicate hydroxide with different morphologies and the comparison of their tribological properties, Tribol Int, 119, pp. 672-9.

18. Gao, K., Chang, Q., Wang, B., Zhou, N., Qing, T., 2018, The tribological performances of modified magnesium silicate hydroxide as lubricant additive, Tribol Int, 121, pp. 64-70.

19. Gulzar, M., Masjuki, H., Varman, M., Kalam, M., Mufti, R.A., Zulkifli, N., et al., 2015, Improving the AW/EP ability of chemically modified palm oil by adding $\mathrm{CuO}$ and $\mathrm{MoS}$ nanoparticles, Tribol Int, 88, pp. 271-9.

20. Chen, S., Liu, W., 2006, Oleic acid capped PbS nanoparticles: Synthesis, characterization and tribological properties, Mater Chem Phys, 98, pp. 183-9.

21. Song, X., Zheng, S., Zhang, J., Li, W., Chen, Q., Cao, B., 2012, Synthesis of monodispersed $\mathrm{ZnAl}_{2} \mathrm{O}_{4}$ nanoparticles and their tribology properties as lubricant additives, Mater Res Bull, 47, pp. 4305-10.

22. Hugh, S., 2015, Friction modifier additives, Tribology Letters, 60, pp. 1-31.

23. Campen, S., Green, J.H., Lamb, G.D., Spikes, H.A., 2015, In Situ Study of Model Organic Friction Modifiers Using Liquid Cell AFM; Saturated and Mono-unsaturated Carboxylic Acids, Tribol Lett, 57, pp. 1-32.

24. Bhushan, B., Liu, H., 2004, Self-assembled monolayers for controlling adhesion, friction and wear, in Bushan, B. (Ed.), Nanotribology and Nanomechanics, Springer, Berlin, Heidelberg, pp. 885-928.

25. Ta, D.T., Tieu, A.K., Zhu, H.T., Kosasih, B., 2015, Thin film lubrication of hexadecane confined by iron and iron oxide surfaces: A crucial role of surface structure, J Chem Phys, 143, 164702.

26. Loehle, S., Matta, C., Minfray, C., Mogne, T., Le Martin, J.M., Iovine, R., et al., 2014, Mixed lubrication with C18 fatty acids: Effect of unsaturation, Tribol Lett, 53, pp. 319-28.

27. Loehlé, S., Matta, C., Minfray, C., Mogne, T. Le, Iovine, R., Obara, Y., et al., 2015, Mixed lubrication of steel by C18 fatty acids revisited. Part I: Toward the formation of carboxylate, Tribol Int, 82, pp. 218-27.

28. Ewen, J.P., Echeverri Restrepo, S., Morgan, N., Dini, D., 2017, Nonequilibrium molecular dynamics simulations of stearic acid adsorbed on iron surfaces with nanoscale roughness. Tribol Int,107, pp. 264-73.

29. Doig, M., Warrens, C.P., Camp, P.J., 2014, Structure and friction of stearic acid and oleic acid films adsorbed on iron oxide surfaces in squalane, Langmuir, 30, pp. 186-95. 